\title{
Avaliação genética de tilápias-do-nilo durante cinco anos de seleção
}

\author{
Carlos Antonio Lopes de Oliveira(1), Grazyella Massako Yoshida(1), Sheila Nogueira de Oliveira(1), \\ Natali Miwa Kunita(1), Alexandra Inês dos Santos ${ }^{(2)}$, Luiz Alexandre Filho ${ }^{(1)}$ e Ricardo Pereira Ribeiro(1)
}

(1)Universidade Estadual de Maringá, Departamento de Zootecnia, Avenida Colombo, № 5.790, Jardim Universitário, CEP 87020-900 Maringá, PR, Brasil. E-mail: caloliveira@uem.br, grazyoshida@hotmail.com, she_uem@hotmail.com, nat_kunita@hotmail.com, lafilho@uem.br, rpribeiro@uem.br (2)Universidade Federal da Bahia, Escola de Medicina Veterinária e Zootecnia, Avenida Adhemar de Barros, Ondina, CEP 40170-110 Salvador, BA, Brasil. E-mail: alexandrainess@gmail.com

\begin{abstract}
Resumo - O objetivo deste trabalho foi estimar parâmetros genéticos e valores genéticos das características peso à despesca e ganho de peso diário de tilápias-do-nilo (Oreochromis niloticus), avaliadas durante cinco anos de cultivo em tanques-rede. O conjunto de dados continha registros de 6.650 animais, com valores genéticos preditos de 8.590 animais na matriz de parentesco. O número de animais avaliados foi de 2.196 (33 famílias), em 2008; 1.721 (58 famílias), em 2009; e 2.733 (78 famílias), em 2010. Análises uni- e bicaracter do conjunto de dados foram realizadas com uso do método de inferência bayesiana, por meio do programa computacional MTGSAM. As estimativas de herdabilidade obtidas foram de média magnitude: 0,273 , para ganho de peso diário; e 0,282 , para peso à despesca. A participação dos efeitos de ambiente comum na variação total foi de $15 \%$ para larvicultura e de 5\% para alevinagem. As correlações genéticas e fenotípicas foram de $95 \%$, o que indica forte associação entre as características. O ganho genético foi de 3,8\% por geração, com acumulado de $15 \%$ em quatro gerações. Houve progresso genético e mudanças, causadas pela seleção de tilápias-do-nilo, nas condições de cultivo avaliadas. A seleção quanto ao ganho de peso permite ganhos indiretos relacionados ao peso.
\end{abstract}

Termos para indexação: Oreochromis niloticus, herdabilidade, seleção indireta, tendências genéticas.

\section{Genetic evaluation of Nile tilapia in a five-year selection}

Abstract - The objective of this work was to estimate genetic parameters and breeding values for body weight at harvest and daily weight gain of Nile tilapia (Oreochromis niloticus), evaluated in cages for five years. The data set contained records of 6,650 animals, with predicted breeding values of 8,590 animals in the relationship matrix. The number of animals evaluated was 2,196 (33 families) in 2008; 1,721 (58 families) in 2009; and 2,733 (78 families) in 2010. Single and two-trait analyses from the data set were performed using Bayesian inference methodology by the computer program MTGSAM. The obtained heritability estimates were of medium magnitude, as follows: 0.273 , for daily weight gain; and 0.282 , for body weight at harvest. The participation of common environmental effects in the total variation was of $15 \%$ for larvae and $5 \%$ for nurseries. The genetic and phenotypic correlations were of $95 \%$, indicating a strong association between the traits. Genetic gain was 3.8\% per generation, with accumulated $15 \%$ over four generations. There were genetic progress and changes caused by the selection of Nile tilapia in the evaluated growing conditions. The selection for weight gain allows of indirect gains associated to weight.

Index terms: Oreochromis niloticus, heritability, indirect selection, genetic trends.

\section{Introdução}

O melhoramento genético de espécies aquícolas iniciou-se na década de 1970 com salmão e truta (Gall \& Gross, 1978; Gjoen \& Bentsen, 1997; Gjedrem, 2012). Altos valores de herdabilidade, para as características de importância econômica, combinados à grande fecundidade e ao curto intervalo de gerações (Gjedrem, 2012) permitem ganhos genéticos de 10 a $20 \%$ por geração taxa de crescimento, valores de cinco a seis vezes maiores do que os de espécies terrestres (Gjedrem \& Baranski, 2009).
Apesar do alto potencial de ganho genético das espécies aquícolas, o melhoramento genético de peixes tem progredido pouco. Calcula-se que, no ano de 2010 , no máximo $10 \%$ da produção aquícola foi baseada em estoques melhorados (Gjedrem et al., 2012). A única exceção é o salmão-do-atlântico, cuja produção proveniente de estoques melhorados geneticamente foi de $100 \%$ na Noruega (Gjerde et al., 2007) e de $97 \%$ no mundo todo, no ano de 2002 (Gjedrem, 2004; Gjedrem \& Baranski, 2009).

A maior parte dos programas de seleção genética de tilápias tem-se concentrado em desenvolver animais 
melhorados em sistemas extensivos ou semi-intensivos de produção (Ponzoni et al., 2005; Charo-Karisa et al., 2007; Rezk et al., 2009). No entanto, o grande potencial hídrico e a produção crescente de tilápias no Brasil (Anúario Brasileiro da Pesca e Aquicultura, 2014) favorecem a utilização de animais melhorados em condições de criação intensiva, para suprir a demanda comercial, assim como a utilização de objetivos de seleção voltados para as características de maior interesse comercial, como ganho de peso (Kunita et al., 2013; Yoshida et al., 2013a), peso (Reis Neto et al., 2014) e rendimento de filé (Gjerde et al., 2012; Nguyen et al., 2010).

O melhoramento genético de tilápias no Brasil teve inicio com a importação de 30 famílias da variedade "genetically improved farmed tilapias (GIFT)" provindas da Malásia. O estabelecimento deste núcleo de melhoramento permitiu a realização de estudos para a avaliação genética, com utilização de métodos quantitativos com controle individual de pedigree e seleção, sob as condições ambientais do país (Santos et al., 2011).

Desde então, ganhos genéticos contínuos, bem como ganhos nos setores produtivos vêem sendo observados em animais melhorados no Brasil. Estimativas de herdabilidade quanto ao peso, entre 0,11 e 0,29 , foram encontrados por Santos et al. (2011), Kunita et al. (2013), Yoshida et al. (2013b) e Reis Neto et al. (2014). Quanto à característica volume corporal, o ganho genético de 5,6\% foi relatado por Reis Neto et al. (2014) em trabalho sobre três gerações de seleção. Oliveira et al. (2012) verificaram a redução de 21 dias do período de cultivo de tilápias em tanques-rede, em que utilizaram animais melhorados geneticamente; os mesmos autores observaram rendimento de filé de $38 \%$, em animais avaliados em tanques-rede, no ano de 2010. Com esses resultados, os autores mostram o impacto do processo de avaliação e seleção, realizadas nas condições brasileiras de cultivo, e evidenciam a importância da disseminação de material geneticamente superior para a cadeia produtiva.

O objetivo deste trabalho foi estimar parâmetros genéticos e valores genéticos das características de peso à despesca e ganho de peso diário de tilápias-do-nilo, avaliadas durante cinco anos de cultivo em tanques-rede.

\section{Material e Métodos}

O conjunto de dados utilizados nas análises continha informações sobre o peso à despesca (peso) e o ganho de peso diário (GPD) de tilápias-do-nilo, Oreochromis niloticus (Linnaeus, 1758), cultivadas em sistema de tanques-rede, no período de 2007-2010.

Utilizaram-se informações sobre tilápias-do-nilo produzidas a partir do acasalamento de animais importados da Malásia no ano de 2005, da variedade GIFT, produzidas pelo World Fish Center. Os detalhes do processo inicial de formação das famílias estão descritos por Santos et al. (2011).

As avaliações genéticas e a seleção dos animais permitiram a substituição total do grupo de reprodutores anualmente, o que caracteriza a formação de gerações discretas. Para a produção dos animais avaliados, utilizou-se um sistema de acasalamento hierárquico um macho para duas fêmeas -, em que se utilizaram 180 reprodutores anualmente.

A reprodução e a alevinagem foram realizadas na Estação de Piscicultura, da Universidade Estadual de Maringá (UEM) e da Companhia de Desenvolvimento Agropecuário do Paraná (Codapar), em Floriano, PR, em sistema de hapas (tanques-redes) individuais de acasalamento instaladas em tanques escavados.

A estação de acasalamento correspondeu ao período de novembro a fevereiro em todos os anos de avaliação. As observações de sinais externos e as verificações de desovas e foram feitas duas vezes por semana. Quando observados os sinais iminentes, o macho era colocado no hapa da fêmea e era retirado quando ocorria a desova.

Após a eclosão, as larvas eram mantidas com as mães até o final da estação de reprodução, quando então eram separadas em dois grupos de 50 representantes de cada família e distribuídas, aleatoriamente, em hapas dispostas no tanque de alevinagem.

Ao atingirem peso superior a $10 \mathrm{~g}$, os animais eram identificados por meio de microchips implantados na cavidade visceral ("passive integrated transponder") e, uma semana depois, eram transferidos para o sistema de cultivo em tanques-redes no Rio do Corvo, Município de Diamante do Norte, PR. Os períodos de criação coincidiram com o final do outono, inverno e começo da primavera, o que corresponde a aproximadamente sete meses de cultivo. Para a utilização de diferentes tanques-redes foi necessária a distribuição de forma igual dos representantes das diferentes famílias em cada tanque disponível.

O conjunto de dados continha registros de 6.650 animais, com valores genéticos preditos de 8.590 
animais na matriz de parentesco. O número de animais avaliados foi de: 2.196 (33 famílias), em 2008; 1.721 (58 famílias), em 2009; e 2.733 (78 famílias), em 2010.

Análises unicaracter e bicaracter foram realizadas quanto às características peso e ganho de peso diário (GPD). Na estimação dos componentes de variância e covariância e dos parâmetros genéticos, para as duas características, utilizou-se o seguinte modelo animal, $\mathrm{y}=\mathrm{X} \beta+\mathrm{Z}_{1} \mathrm{a}+\mathrm{Z}_{2} \mathrm{c}+\mathrm{Z}_{3} \mathrm{p}+\varepsilon$, em que: y é o vetor de observações das características analisadas; $\beta$ é o vetor dos efeitos ambientais identificáveis; a, c, p, e $\varepsilon$ são, respectivamente, os vetores dos efeitos genéticos aditivos diretos, efeito comum de ambiente materno de larvicultura, efeito comum de ambiente de alevinagem e dos erros aleatórios. As variáveis $X, Z_{1}, Z_{2}$ e $Z_{3}$ são, as matrizes de incidência respectivas aos seguintes efeitos: ambientais identificáveis; genéticos aditivos diretos; de larvicultura; e comum de ambiente materno de alevinagem. Os efeitos ambientais identificáveis, considerados nas análises, foram: sexo, anos de cultivo, tanques-redes e, como covariáveis, a idade e o número de alevinos.

Para estimar os componentes de variâncias e covariâncias e dos parâmetros genéticos das características, bem como para predizer os valores genéticos dos animais para cada característica, utilizaram-se procedimentos bayesianos, com uso do sistema computacional MTGSAM ("multiple trait Gibbs sampling to animal model"), conforme Van Tassell \& Van Vleck (1998). Os intervalos de credibilidade - a 95\% de probabilidade das estimativas dos componentes de variância e covariância e dos parâmetros genéticos -, foram estimados por meio do sistema R.

Nas análises unicarater, utilizou-se a estratégia de cadeias de Gibbs oriundas de 500 mil ciclos, com período de descarte de 10 mil ciclos e intervalo de retirada de 15 ciclos. Para a análise bicaracter, utilizou-se uma cadeia de um milhão de ciclos, com período de descarte de 100 mil ciclos e intervalo de retirada de 20 ciclos. A convergência das cadeias de Gibbs foi testada por meio do método Heidelberger e Welch, descrito por Best et al. (1995), com uso da biblioteca convergence diagnostics and output analysis (CODA) para a cadeia de Markov-Monte Carlo (MCMC) implementada no sistema R (R Foundation for Statistical Computing, Viena. Áustria).
Estimaram-se as correlações de postos, com base nas classificações das famílias cultivadas no ano de 2010, com a média dos valores genéticos preditos para peso e GPD de cada família. Estimou-se também a correlação existente entre as médias dos valores genéticos preditos para cada família.

Para descrever o comportamento dos valores genéticos, nos diferentes anos, estimaram-se as tendências dos valores genéticos com os anos de cultivo, por meio de equações de regressão linear.

\section{Resultados e Discussão}

Houve indicação de convergência para todas as cadeias obtidas nas análises uni- e bicaracter. As estimativas de herdabilidade, para ambas as características, foram de média magnitude, com 0,27 para GPD e 0,28 para peso, sem diferenças entre as estimativas obtidas nas análises uni- e bicaracter (Tabela 1). A partir dos valores de herdabilidade, estimou-se que cerca de $30 \%$ da variabilidade encontrada é de origem genética aditiva. Assim, parte considerável das diferenças observadas entre os animais é resultante de diferenças herdáveis e pode ser explorada por meio de seleção.

Os intervalos de credibilidade para herdabilidade de ambas as características, nas análises uni- e bicaracter, foram coincidentes (Tabela 1). Os limites inferiores apresentaram valores superiores a 0,14 , enquanto os limites superiores não excederam 0,45 , o que indica a precisão das estimativas e que estas são significativas.

Ao estudar tilápias em diferentes ambientes de cultivo, Bentsen et al. (2012) observaram herdabilidades do peso corporal de 0,06 a 0,30 , em animais avaliados em viveiros, e de 0,31 a 0,68 , em animais avaliados em tanques-redes, o que evidencia potencial para o melhoramento em ambos os ambientes. No entanto, os autores citados sugerem que as herdabilidades tendem a ser maiores em populações avaliadas em tanques-redes do que em viveiros, pois, espera-se menor variação ambiental e maturação sexual mais tardia, o que compromete menos o crescimento dos animais em tanques-redes e ressalta a importância da avaliação e seleção dos animais de acordo com o ambiente em que serão cultivados.

Com uso de informações referentes a animais avaliados do programa de melhoramento de tilápias em Maringá, somente no ano de 2008, Santos et al. (2011) encontraram estimativa de herdabilidade de 
0,24 (intervalo de credibilidade de 0,13 a 0,35 ) da característica peso à despesca, em análises unicaracter. Com os registros de 1.817 animais avaliados no ano de 2009, pelo mesmo programa de melhoramento, Yoshida et al. (2013a) obtiveram herdabilidades de 0,11 e 0,12 (uni e bicaracter respectivamente), quanto ao peso, e 0,13 e 0,14 (uni e bicaracter respectivamente) quando ao GPD. As diferenças observadas nas estimativas, entre os valores obtidos no presente trabalho, em comparação aos citados anteriormente, podem ser explicadas pela quantidades diferentes de animais utilizados nas análises e pela utilização de informações de pedigree com maior número de indivíduos. Além disso, foram anos de avaliação diferentes, os animais foram submetidos a condições ambientais referentes ao ano em que foram avaliados.

A participação do efeito de ambiente comum de larvicultura foi inferior a $15 \%$ e não houve estimativas discrepantes entre as análises uni e bicaracter (Tabela 1). Em relação ao efeito de ambiente comum de alevinagem, verificou-se que a importância relativa foi inferior a 5\%, para ambas as características, porém se verificou discrepância nas estimativas deste efeito quanto à característica peso, entre as análises, com subestimação da bicaracter em relação à análise unicaracter.

Quando se considerou o efeito comum de alevinagem, verificou-se menor importância relativa deste efeito na variação total. No entanto, com os intervalos de credibilidade, pode-se inferir que estes valores são diferentes de zero e devem ser considerados no modelo. A manutenção de grupos de irmãos completos, nas mesmas condições ambientais (hapas de alevinagem), acarretou menores diferenças entre o grupo de irmãos completos e aumentou a variabilidade entre as famílias. Os valores das estimativas dos efeitos de ambiente comum de larvicultura na variação total são indicativos da importância deste efeito como causa das diferenças entre os animais. A desconsideração destes efeitos no modelo implicaria na superestimação dos valores de herdabilidade, e diminuiria, assim, a acurácia da avaliação genética.

Em trabalhos realizados com tilápias-do-nilo em tanques-rede, Gjerde et al (2012) e Khaw et al. (2012) encontraram valores de ambiente comum de família de 0,23 e 0,14 , respectivamente, na característica peso à despesca. Em programas de melhoramento de tilápias realizados em outros países, utiliza-se somente um efeito de ambiente comum, referente ao período da eclosão até os animais atingirem o tamanho suficiente para a identificação. No presente trabalho, não houve incubação artificial, os alevinos foram mantidos com as mães até o final da estação de acasalamento, o que resultou em dois ambientes comuns, porém, observase que as estimativas conjuntas destes ambientes apresentaram valores intermediários aos trabalhos citados.

Estimativas com uso de dois efeitos de ambientes comuns foi realizado por Kunita et al. (2013); estes autores obtiveram valores do efeito do ambiente comum de larvicultura de 0,11 e 0,12 quanto ao peso e GPD, respectivamente, e $0,02 \mathrm{em}$ ambas as características para o efeito de alevinagem. Estes resultados são corroborados pelos obtidos no presente trabalho e evidenciam a maior participação do efeito de ambiente comum de larvicultura na variação total. Tal resultado é

Tabela 1. Estimativas de parâmetros genéticos e de variância fenotípica das características peso à despesca e ganho de peso diário, com respectivas médias \pm desvios-padrão e intervalos de credibilidade (IC) a 95\%, em análises unicaracter (uni) e bicaracter (bi).

\begin{tabular}{|c|c|c|c|c|c|}
\hline \multirow[t]{2}{*}{ Parâmetro } & \multirow[t]{2}{*}{ Análise } & \multicolumn{2}{|c|}{ Peso à despesca } & \multicolumn{2}{|c|}{ Ganho de peso diário } \\
\hline & & Média \pm desvio-padrão & IC & Média \pm desvio-padrão & IC \\
\hline \multirow{2}{*}{$h^{2}$} & Unicaracter & $0,282 \pm 0,074$ & $0,153-0,439$ & $0,273 \pm 0,074$ & $0,140-0,431$ \\
\hline & Bicaracter & $0,282 \pm 0,075$ & $0,147-0,439$ & $0,272 \pm 0,073$ & $0,141-0,425$ \\
\hline \multirow{2}{*}{$\mathrm{L}^{2}$} & Unicaracter & $0,138 \pm 0,027$ & $0,089-0,195$ & $0,132 \pm 0,027$ & $0,083-0,189$ \\
\hline & Bicaracter & $0,138 \pm 0,028$ & $0,087-0,196$ & $0,130 \pm 0,027$ & $0,081-0,186$ \\
\hline \multirow{2}{*}{$\mathrm{C}^{2}$} & Unicaracter & $0,049 \pm 0,012$ & $0,028-0,077$ & $0,027 \pm 0,006$ & $0,016-0,041$ \\
\hline & Bicaracter & $0,026 \pm 0,006$ & $0,016-0,041$ & $0,026 \pm 0,006$ & $0,015-0,041$ \\
\hline \multirow{2}{*}{$\sigma_{\mathrm{p}}^{2}$} & Unicaracter & $11.570 \pm 500,9$ & $10.710-12.660$ & $0,193 \pm 0,008$ & $0,179-0,211$ \\
\hline & Bicaracter & $11.550 \pm 495,5$ & $10.680-12.640$ & $0,193 \pm 0,008$ & $0,179-0,210$ \\
\hline
\end{tabular}

$\mathrm{h}^{2}$, herdabilidade; $\mathrm{L}^{2}$ e $\mathrm{C}^{2}$, importância dos efeitos de ambiente comum de larvicultura e de ambiente comum de alevinagem, respectivamente; $\sigma_{\mathrm{P}}^{2}$, variância fenotípica.

Pesq. agropec. bras., Brasília, v.50, n.10, p.871-877, out. 2015

DOI: 10.1590/S0100-204X2015001000002 
um indicativo de que, nas gerações futuras, o emprego da técnica de incubação artificial será necessária para desconsiderar este efeito no modelo e, como consequência, a variação fenotípica será reduzida, o que pode resultar em valores de herdabilidades superiores àquelas já estimadas para o presente programa. Assim, é justificável a utilização de incubação artificial, a identificação dos animais precocemente, a alocação aleatória dos animais no ambiente de alevinagem e um maior controle das variações ambientais, durante esta fase, para reduzir a variação fenotípica e melhorar a acurácia das estimativas. Com estes procedimentos e de acordo com a reduzida importância do ambiente comum de alevinagem já relatado, espera-se que os valores do efeito de ambiente comum, para a população avaliada no Brasil, sejam menores do que as estimativas relatadas na literatura.

As correlações genéticas e fenotípicas estimadas entre as duas características foram 0,988 e 0,972, respectivamente (Tabela 2), o que indica que é possível obter resultados com a resposta correlacionada e, assim, incrementar o peso do animal à despesca, a partir da seleção quanto à velocidade de ganho de peso. Os intervalos de credibilidade destas estimativas foram de 0,975 a 0,994 , para correlação genética, e de 0,966 a 0,975 , para correlação fenotípica, o que indica a existência de forte associação genética e fenotípica entre as características. As pequenas amplitudes dos intervalos de credibilidade confirmam a forte associação e precisão destas estimativas.

Os valores genéticos preditos para os animais, no último ano de cultivo, foram superiores aos dos demais anos (Tabela 2), o que indica superioridade genética da atual geração de animais candidatos à seleção, em

Tabela 2. Estatísticas descritivas dos valores genéticos preditos quanto ao ganho de peso diário (GPD) e peso à despesca (peso), a partir da análise bicaracter.

\begin{tabular}{|c|c|c|c|c|c|}
\hline Ano & Característica & Média & Desvio-padrão & Máximo & Mínimo \\
\hline \multirow{2}{*}{2005} & Peso & $-0,304$ & 7,894 & 24,610 & $-28,979$ \\
\hline & GPD & $-0,001$ & 0,033 & 0,107 & $-0,115$ \\
\hline \multirow{2}{*}{2006} & Peso & $-0,560$ & 8,75 & 53,239 & $-55,237$ \\
\hline & GPD & $-0,002$ & 0,036 & 0,229 & $-0,2164$ \\
\hline \multirow{2}{*}{2008} & Peso & $-1,06$ & 26,203 & 85,49 & $-75,379$ \\
\hline & GPD & $-0,005$ & 0,106 & 0,362 & $-0,301$ \\
\hline \multirow{2}{*}{2009} & Peso & 23,125 & 22,654 & 119,378 & $-44,118$ \\
\hline & GPD & 0,09 & 0,088 & 0,463 & $-0,164$ \\
\hline \multirow{2}{*}{2010} & Peso & 35,607 & 32,953 & 155,295 & $-73,651$ \\
\hline & GPD & 0,141 & 0,131 & 0,615 & $-0,284$ \\
\hline
\end{tabular}

relação aos demais indivíduos das gerações que os antecederam, como resultado dos esforços de seleção para produzir indivíduos geneticamente superiores.

Com as estimativas de tendências genéticas, observou-se incremento dos valores genéticos ao longo dos anos de seleção, com taxas anuais de mudanças de 0,0528 g por dia e 13,663 g por período de cultivo, para GPD e peso, respectivamente. Com base nestes valores, estimaram-se os ganhos genéticos anuais de $3,8 \%$, para ambas as características e ganhos genéticos acumulados de $15 \%$ em quatro gerações.

As estimativas de ganhos genéticos, obtidas no presente trabalho quanto ao peso à despesca, foram inferiores aos $8 \%$ por geração durante seis anos de seleção (Thodesen et al., 2012), e aos 11,4\%, em cinco gerações de seleção (Nguyen et al., 2010). Com informações somente de uma geração do programa de melhoramento genético de tilápias, realizado no Estado do Paraná, Santos et al. (2011) encontraram ganhos genéticos de 5,07\% para peso à despesca e de 2,0\% para ganho indireto para sobrevivência. Estimativas discrepantes em diferentes trabalhos podem ser encontradas em decorrência da maior ou menor intensidade de seleção aplicada, que tendem a se reduzir em gerações posteriores, para produzir as famílias em intervalo menor de tempo e, para isto, é necessário aumentar até em três vezes o número de fêmeas selecionadas (Thodesen et al., 2012).

Verificou-se que não houve alterações dos valores de correlação de Spearman e Pearson, nas diferentes gerações e, nem com uso de informações de todas as gerações (Tabela 3). Tais estimativas, preditas entre GPD e peso, são indicativas de que as avaliações genéticas quanto ao peso vivo e ao ganho de peso diário conduzem a seleção do mesmo conjunto de indivíduos. Assim, a seleção quanto à velocidade de crescimento resulta em incremento do peso dos animais.

Tabela 3. Correlações de Pearson e de Spearman dos valores genéticos entre as características peso à despesca e ganho de peso diário.

\begin{tabular}{lccc}
\hline Ano & $\begin{array}{c}\text { Correlação de } \\
\text { Pearson }\end{array}$ & $\begin{array}{c}\text { Correlação de } \\
\text { Spearman }\end{array}$ & $\begin{array}{c}\text { Valor genético } \\
\text { predito }\end{array}$ \\
\hline 2005 & 0,997 & 0,985 & 86 \\
2006 & 0,997 & 0,984 & 1527 \\
2008 & 0,998 & 0,998 & 2258 \\
2009 & 0,996 & 0,996 & 1729 \\
2010 & 0,995 & 0,996 & 2990 \\
\hline Total & 0,997 & 0,996 & 8590 \\
\hline
\end{tabular}




\section{Conclusões}

1. Há ganhos genéticos significativos em cinco anos de seleção de tilápias-do-nilo.

2. A seleção quanto ao ganho de peso permite ganhos indiretos relacionados a outros caracteres.

3. A magnitude das herdabilidades sustentam o progresso genético para as próximas gerações.

\section{Agradecimentos}

Ao Conselho Nacional de Desenvolvimento Científico e Tecnológico (CNPq), pelo apoio financeiro; e aos funcionários e alunos da Estação Experimental de Piscicultura, da Universidade Estadual de Maringá, pela ajuda nas coletas.

\section{Referências}

ANÚARIO BRASILEIRO DA PESCA E AQUICULTURA. Rio de Janeiro: ACEB, v.1, 2014. 133p.

BENTSEN, H.B.; GJERDE, B.; NGUYEN, N.H.; RYE, M.; PONZONI, R.W.; DE VERA, M.S.P.; BOLIVAR, H.L.; VELASCO, R.R.; DANTING, J.C.; DIONISIO, E.E.; LONGALONG, F.M.; REYES, R.A.; ABELLA, T.A.; TAYAMEN, M.M.; EKNATH, A.E. Genetic improvement of farmed tilapias: genetic parameters for body weight at harvest in Nile tilapia (Oreochromis niloticus) during five generations of testing in multiple environments. Aquaculture, v.338-341, p.56-65, 2012. DOI: 10.1016/j. aquaculture.2012.01.027.

BEST, N.; COWLES, M.K.; VINES, K. CODA. Convergence diagnosis and output analysis software for Gibbs sampling output. Verision 0.30. Cambridge: Institute of Public Health, MRC Biostatistics Unit, 1995.

CHARO-KARISA,H.;BOVENHUIS,H.;REZK,M.A.;PONZONI, R.W.; VAN ARENDONK, J.A.M.; KOMEN, H. Phenotypic and genetic parameters for body measurements, reproductive traits and gut length of Nile tilapia (Oreochromis niloticus) selected for growth in low-input earthen ponds. Aquaculture, v.273, p.15-23, 2007. DOI: 10.1016/j.aquaculture.2007.09.011.

GALL, G.A.E.; GROSS, S.J. Genetic studies of growth in domesticated rainbow trout. Aquaculture, v.13, p.225-234, 1978. DOI: 10.1016/0044-8486(78)90004-2.

GJOEN, H.M.; BENTSEN, H.B. Past, present, and future of genetic improvement in salmon aquaculture. ICES Journal of Marine Science, v.54, p.1009-1014, 1997. DOI: 10.1016/ S1054-3139(97)80005-7.

GJEDREM, T. Genetic improvement for the development of efficient global aquaculture: a personal opinion review. Aquaculture, v.344, p.12-22, 2012. DOI: 10.1016/j.aquaculture.2012.03.003.

GJEDREM, T. Status for breeding programs in aquaculture. In: FISH BREEDER'S ROUNDTABLE, 2004, Håholmen, Norway. [Proceedings] Tromsø: Nofima, 2004.
GJEDREM, T.; BARANSKI, M. Selective breeding in aquaculture: an introduction. Dordrecht: Springer, 2009. 221p. (Methods and technologies in fish biology and fisheries, 10). DOI: 10.1007/978-90-481-2773-3.

GJEDREM, T.; ROBINSON, N.; RYE, M. The importance of selective breeding in aquaculture to meet future demands for animal protein: a review. Aquaculture, v.350-353, p.117-129, 2012. DOI: 10.1016/j.aquaculture.2012.04.008.

GJERDE, B.; MENGISTU, S.B.; ØDEGÅRD, J.; JOHANSEN, H.; ALTAMIRANO, D.S. Quantitative genetics of body weight, fillet weight and fillet yield in Nile tilapia (Oreochromis niloticus). Aquaculture, v.342, p.117-124, 2012. DOI: 10.1016/j. aquaculture.2012.02.015.

GJERDE, B.; SONESSON, A.; STORSET, A.; RYE, M. Selective breeding and genetics: Atlantic salmon. In: THOMASSEN, M.; GUDDING, R.; NORBERG, B.; JØRGENSEN, L. Aquaculture research: from cage to consumer. Oslo: The Research Council of Norway, 2007. p.268-284

KHAW, H.L.; PONZONI, R.W.; HAMZAH, A.; ABU-BAKAR, K.R.; BIJMA, P. Genotype by production environment interaction in the GIFT strain of Nile tilapia (Oreochromis niloticus). Aquaculture, v.326-329, p.53-60, 2012. DOI: 10.1016/j. aquaculture.2011.11.016.

KUNITA, N.M.; OLIVEIRA, C.A.L.; OLIVEIRA, S.N.; YOSHIDA, G.M.; RIZZATO, G.S.; RESENDE, E.K.; RIBEIRO, R.P. Avaliação genética de características morfométricas em tilápias do Nilo cultivadas. Archivos de Zootecnia, v.62, p.555-566, 2013. DOI: $10.4321 /$ S0004-05922013000400008.

NGUYEN, N.H.; PONZONI, R.W.; ABU-BAKAR, K.R.; HAMZAH, A.; KHAW, H.L.; YEE, H.Y. Correlated response in fillet weight and yield to selection for increased harvest weight in genetically improved farmed tilapia (GIFT strain), Oreochromis niloticus. Aquaculture, v.305, p.1-5, 2010. DOI: 10.1016/j. aquaculture.2010.04.007.

OLIVEIRA, C.A.L.; RIBEIRO, R.P.; STREIT JUNIOR, D.; POVH, J.A.; RESENDE, E.K. Melhoramento genético de peixes: uma realidade para a piscicultura brasileira. Panorama da Aquicultura, v.22, p.38-47, 2012.

PONZONI, R.W.; HAMZAH, A.; TAN, S.; KAMARUZZAMAN, $\mathrm{N}$. Genetic parameters and response to selection for live weight in the GIFT strain of Nile Tilapia (Oreochromis niloticus). Aquaculture, v.247, p.203-210, 2005. DOI: 10.1016/j.aquaculture.2005.02.020.

REIS NETO, R.V.; OLIVEIRA, C.A.L. de; RIBEIRO, R.P.; FREITAS, R.T.F. de; ALLAMAN, I.B.; OLIVEIRA, S.N. de. Genetic parameters and trends of morphometric traits of GIFT tilapia under selection for weight gain. Scientia Agricola, v.71, p.259-265, 2014. DOI: 10.1590/0103-9016-2013-0294.

REZK, M.A.; PONZONI, R.W.; KHAW, H.L.; KAMEL, E.; DAWOOD, T.; JOHN, G. Selective breeding for increased body weight in a synthetic breed of Egyptian Nile tilapia, Oreochromis niloticus: response to selection and genetic parameters. Aquaculture, v.293, p.187-194, 2009. DOI: 10.1016/j. aquaculture.2009.03.019.

SANTOS, A.I.; RIBEIRO, R.P.; VARGAS, L.; MORA, F.; ALEXANDRE FILHO, L.; FORNARI, D.C.; OLIVEIRA, S.N. de. 
Bayesian genetic parameters for body weight and survival of Nile tilapia farmed in Brazil. Pesquisa Agropecuária Brasileira, v.46, p.33-43, 2011. DOI: 10.1590/S0100-204X2011000100005.

THODESEN, J.; RYE, M.; WANG, Y.X.; BENTSEN, H.B.; GJEDREM, T. Genetic improvement of tilapias in China: genetic parameters and selection responses in fillet traits of Nile tilapia (Oreochromis niloticus) after six generations of multi-trait selection for growth and fillet yield. Aquaculture, v.366-367, p.67-75, 2012. DOI: 10.1016/j.aquaculture.2011.10.010.

VAN TASSELL, C.P.; VAN VLECK, L.D.; GREGORY, K.E. Bayesian analysis of twinning and ovulation rates using a multiple-trait threshold model and Gibbs sampling. Journal of Animal Science, v.76, p.2048-2061, 1998.
YOSHIDA, G.M.; OLIVEIRA, C.A.L.; KUNITA, N.M.; OLIVEIRA, S.N.; ALEXANDRE FILHO, L.; RESENDE, E.K.; LOPERA-BARRERO, N.M.; RIBEIRO, R.P. Resposta à seleção de características de desempenho e morfométricas de tilápias-do-nilo ao longo do período de cultivo. Arquivo Brasileiro de Medicina Veterinária e Zootecnia, v.65, p.1815-1822, 2013b. DOI: 10.1590/ S0102-09352013000600032.

YOSHIDA, G.M.; OLIVEIRA, C.A.L.; OLIVEIRA, S.N.; KUNITA, N.M.; RESENDE, E.K.; ALEXANDRE FILHO, L.; RIBEIRO, R.P. Associação entre características de desempenho de tilápia-do-nilo ao longo do período de cultivo. Pesquisa Agropecuária Brasileira, v.48, p.816-824, 2013a. DOI: 10.1590/ S0100-204X2013000800002.

Recebido em 8 de abril de 2015 e aprovado em 21 de julho de 2015 